\title{
Abnormalities in the ultradian oscillations of insulin secretion and glucose levels in Type 2 (non-insulin-dependent) diabetic patients
}

\author{
J.Sturis $^{1,2}$, K.S.Polonsky ${ }^{1}$, E. T.Shapiro ${ }^{1}$, J.D. Blackman ${ }^{1}$, N.M. O'Meara ${ }^{1}$ and E. Van Cauter ${ }^{1,3}$ \\ ${ }^{1}$ Department of Medicine, University of Chicago, Chicago, Illinois, USA, \\ ${ }^{2}$ Physics Laboratory III, Technical University of Denmark, Lyngby, Denmark, and \\ ${ }^{3}$ Institute of Interdisciplinary Research, Free University of Brussels, Brussels, Belgium
}

\begin{abstract}
Summary. To investigate the temporal organization of insulin secretion and glucose concentration during fasting in Type 2 (non-insulin-dependent) diabetes mellitus, we studied seven patients with Type 2 diabetes, eight obese non-diabetic control subjects and eight normal weight non-diabetic subjects. Blood sampling for glucose, insulin and C-peptide was performed at 15 -min intervals during a $24-\mathrm{h}$ period of fasting for the diabetic and the obese control subjects and during an 8-h fasting period for the normal subjects. Insulin secretion rates were calculated from the peripheral C-peptide concentration profiles. Ultradian oscillations of glucose levels and insulin secretion rates were evident during fasting in all subjects. An additional study with blood sampling at 2-min intervals for $8 \mathrm{~h}$ further indicated that this ultradian periodicity is expressed independently of rapid $10-15 \mathrm{~min}$ insulin oscillations. There were no differences between diabetic and nondiabetic subjects in the frequency of the ultradian oscillations of insulin secretion (which averaged 12-15 oscillations per $24 \mathrm{~h}$ ) and in the rate of concomitancy of oscillations of insulin
\end{abstract}

secretion with oscillations in glucose levels, which averaged $63-65 \%$. The relative amplitudes of both the insulin and glucose oscillations were also similar in diabetic and nondiabetic subjects. The major abnormality in patients with Type 2 diabetes was evidenced by spectral analysis, and confirmed by calculations of the distributions of inter-pulse intervals. It consisted of a slowing of the glucose oscillations, without a similar slowing of the oscillations in insulin secretion. This slowing of the glucose oscillations in fasting Type 2 diabetic patients is consistent with our previous observations of sluggish and irregular glucose oscillations in diabetic subjects receiving mixed meals. This partial dissociation between the oscillatory patterns of insulin secretion and glucose levels could represent a sensitive quantitative marker of the breakdown of the insulin-glucose feedback loop in diabetes.

Key words: Ultradian oscillations, insulin secretion, glucose, spectral analysis, pulse, temporal pattern.
The temporal organization of Beta-cell secretion is complex and includes "oscillatory" or "pulsatile" activity in two different period ranges. Rapid secretory oscillations with a periodicity of approximately $10-15 \mathrm{~min}[1,2]$ as well as slower ultradian oscillations recurring at intervals of 80-140 min have been described [3-7]. The ultradian oscillations in glucose and insulin concentrations and in insulin secretion rate (ISR) have been observed in a variety of conditions in which pancreatic insulin secretion was stimulated including constant glucose infusion $[5,7]$, continuous enteral nutrition [4], meal ingestion $[6,8,9]$ and oral glucose loads [3]. It remains to be determined whether ultradian oscillatory behaviour also exists during non-stimulated conditions or only characterizes the hyperglycaemic state. During overnight fasting following a mixed meal, we have previously noted the occurrence of infrequent, low amplitude pulses of insulin secretion $[6,9]$. However, in these previous studies, the frequency of blood sampling and the duration of the fasting period were insufficient to determine whether these sporadic small pulses reflected ultradian oscillatory behaviour or were caused by an undersampling of the rapid 10-15 min oscillations.

The mechanism underlying the generation of the ultradian oscillations remains to be elucidated. Although it has been speculated that they may be due to the activity of a glucose-independent intrapancreatic pacemaker [10], the recent demonstration that, in normal subjects, they can be completely entrained by oscillatory infusions of exogenous glucose [11] supports the hypothesis that glucose is an important part of the generating mechanism. This hypothesis is supported by the results of extensive simulations of a mathematical model of insulin-glucose interactions which indicate that such oscillations could originate from the negative feedback interactions between insulin secretion and glucose concentrations [12]. This model further 
predicts that the ultradian oscillations will be progressively dampened during fasting.

In patients with untreated Type 2 (non-insulin-dependent) diabetes mellitus, the pattern of oscillations in glucose and ISR which occur following meal ingestion is profoundly altered [9]. Indeed, the amplitude of the pulses is less than half that observed in non-diabetic control subjects. Furthermore, while in normal control subjects, 80 $90 \%$ of the oscillations of insulin secretion occur in association with a pulse in plasma glucose, in Type 2 diabetic patients, the proportion of pulses which occur in temporal association with a glucose pulse is reduced to about $50 \%$ [9]. The pattern of oscillations also appears to be less regular. These observations have raised the possibility that the abnormal ultradian oscillations may have pathogenetic significance in Type 2 diabetes. It is not known whether abnormalities in ultradian oscillations which are evident postprandially may also be detected during prolonged fasting, when glucose levels are markedly reduced. Disruptions of the oscillatory pattern were less pronounced after breakfast than after the evening meal [9], suggesting that the overnight fasting period is of some restorative value. However, the persistence of abnormalities in ultradian oscillations in fasting Type 2 diabetic patients would suggest that they may constitute a sensitive early marker of the disorder.

The present study was undertaken to determine whether ultradian oscillations of insulin secretion and glucose levels persist under non-stimulated conditions and whether abnormalities associated with the diabetic state can be detected even when glucose levels are reduced by prolonged fasting. Profiles of plasma glucose and ISR obtained at 15 -min intervals during a 24 -h fast were compared in patients with Type 2 diabetes and non-diabetic obese subjects matched for age, sex, and body weight. Normal subjects were similarly studied during an 8-h fast. In addition, to determine the relationship between the ultradian oscillations and the rapid $10-15$ min oscillations, profiles of plasma glucose, plasma C-peptide and serum insulin were obtained by sampling at 2-min intervals for $8 \mathrm{~h}$.

\section{Subjects and methods}

\section{Subjects}

Seven patients with Type 2 diabetes (three men and four women) and eight obese non-diabetic control subjects (three men and five women) were studied. The diabetic patients and the control subjects were well matched for age (Type 2 diabetic patients $51 \pm 4$ years, range $35-69$ years; obese control subjects $48 \pm 3$ years, range 40-64 years) and body mass index (Type 2 diabetic patients $34.8 \pm 2.1 \mathrm{~kg} / \mathrm{m}^{2}$, range $31.2-42.3 \mathrm{~kg} / \mathrm{m}^{2}$; obese control subjects $33.0 \pm 2.8 \mathrm{~kg} / \mathrm{m}^{2}$, range $25.6-49.2 \mathrm{~kg} / \mathrm{m}^{2}$ ). An analysis of the diurnal changes in glucose concentrations and insulin secretion and of their relationship to patterns of counterregulatory hormones in the Type 2 diabetic patients and their control subjects has been previously published [13]. In addition, nine normal subjects within $20 \%$ of ideal body weight (BMI $22.4 \pm 0.9 \mathrm{~kg} / \mathrm{m}^{2}$ ) were studied. All obese control and lean subjects had normal fasting glucose levels as well as a negative family history of diabetes.
Patients with diabetes were being treated with either oral hypoglycaemic agents (three of seven) or diet (all seven) or both. Oral hypoglycaemic agents were discontinued at least 2 weeks prior to the study. None of the patients had undergone prior therapy with insulin. The mean duration of diagnosed diabetes was $4.7 \pm 1.2$ years.

All studies were carried out in the Clinical Research Center of the University of Chicago after written informed consent had been obtained. The experimental protocols were approved by the Institutional Review Board.

\section{Experimental Protocol}

1. Temporal profiles of glucose and ISR during fasting. Following a 10 -h overnight fast, the diabetic patients and their matched control subjects fasted for an additional $24 \mathrm{~h}$ beginning and ending at 08.00 hours. Following a $10-\mathrm{h}$ overnight fast, the normal subjects fasted for $8 \mathrm{~h}$, beginning at 08.00 hours and ending at 16.00 hours. During the fasting period, the subjects remained in a recumbent position. They were not allowed any food but had free access to water. An i.v. sampling catheter was inserted into a dorsal vein of one hand. This hand was kept in a heating blanket to ensure arterialization of the blood samples. Starting at 08.00 hours, blood samples for the measurement of glucose, insulin and C-peptide were obtained at 15 -min intervals for the duration of each study in the diabetic patients, their control subjects and the normal subjects.

2. Derivation of $C$-peptide kinetics. The parameters quantifying Cpeptide clearance kinetics were obtained on a separate occasion in all the volunteers who participated in the study described. After a 10 -h overnight fast, an $i$. v. sampling catheter was inserted into a dorsal vein of one hand and an infusion catheter was inserted into the opposite hand. Endogenous C-peptide secretion was inhibited by means of a primed infusion of somatostatin $(500 \mu \mathrm{g} / \mathrm{h}$, Bachem Fine Chemicals, Torrance, Calif., USA). One hour after initiation of the somatostatin infusion, an $\mathrm{i}$. v. bolus injection of $150 \mu \mathrm{g}$ biosynthetic human C-peptide (Eli Lilly and Co., Indianapolis, Ind., USA) was given to each subject. Plasma $\mathrm{C}$-peptide levels were measured frequently over the following $180 \mathrm{~min}$. The kinetic parameters of C-peptide distribution and metabolism were determined for each subject according to a two-compartment model as previously described $[14,15]$. The volume of distribution was larger in the diabetic subjects $(4766 \pm 418 \mathrm{ml})$ and in the obese control subjects $(4306 \pm 213 \mathrm{ml})$ than in the normal subjects $(3371 \pm 164 \mathrm{ml}$, $p<0.05$ ). The long half-life (Type 2: $31.8 \pm 3.6 \mathrm{~min}$; obese: $38.3 \pm 2.3 \mathrm{~min}$; normal: $30.0 \pm 1.8 \mathrm{~min}$ ), short half-life (Type 2: $3.58 \pm 0.45 \mathrm{~min}$; obese: $4.90 \pm 0.56 \mathrm{~min}$; normal: $4.84 \pm 0.02 \mathrm{~min}$ ) and fraction associated with the short half-life (Type 2: $0.769 \pm 0.009$; obese: $0.758 \pm 0.013$; normal: $0.780 \pm 0.020$ ) did not differ significantly across the three groups. For each subject, the model parameters were derived algebraically from these kinetic parameters and were used to derive the insulin secretory rates from the peripheral C-peptide levels measured during the $2 \mathrm{~A}$-h fasting study by deconvolution as previously described [15].

3. Rapid sampling study. Following a 10 -h overnight fast, an additional normal subject was studied for an 8 -h period, starting at 08.00 hours. The subject remained fasted and in a recumbent position throughout the study. Blood samples for the measurement of plasma glucose, plasma C-peptide and serum insulin were taken at 2-min intervals for the duration of the study.

\section{Glucose, insulin and C-peptide assays}

Glucose concentrations were measured by a glucose analyser (Yellow Springs Instrument Co., Model 23A, Yellow Springs, Ohio, USA). In the present studies, the coefficient of variation of replicate determinations of plasma glucose levels obtained by this method was below $1.5 \%$. Serum insulin levels were determined by a double anti- 
body technique [16] with a lower level of sensitivity of $20 \mathrm{pmol} / \mathrm{h}$ and an intra-assay coefficient of variation of $6 \%$. Plasma C-peptide levels were measured as previously described [17]. The lower limit of sensitivity of the assay is $20 \mathrm{pmol} / \mathrm{l}$. The intra-assay coefficient of variation averaged $4 \%$. All samples from the same subject were analysed in the same assay.

\section{Data analysis}

Smoothing. Except for the profiles obtained with the rapid sampling frequency, the individual time series for glucose, insulin and C-peptide were smoothed using a two-point moving average. This procedure consists of replacing the value observed at time $t$ by the arithmetic mean of the values observed at times $t-\Delta$ and $t$ where $\Delta$ is the sampling interval, i.e. $15 \mathrm{~min}$. Smoothing with a two-point moving average strongly dampens fluctuations shorter than $30 \mathrm{~min}$ while only modestly reducing the amplitude of slower variations. After smoothing with a two-point moving average, the measurement error is reduced by a factor $\sqrt{2}$. All further calculations were performed on the smoothed profiles. The smoothed C-peptide profiles were used to derive ISR by deconvolution.

Detrending. As expected, during fasting, glucose levels, insulin levels and ISR exhibited a declining trend which partially masked the occurrence of more rapid changes. To remove these long-term trends and optimize the process of pulse identification, a best-fit curve was calculated for each smoothed glucose, insulin, and ISR profile using the robust non-linear regression procedure proposed by Cleveland [18] with a window of $8 \mathrm{~h}$. This best-fit curve was then subtracted from the data and replaced by the mean level of all data collected during the fasting period. The resulting profiles will be referred to as the detrended series. All subsequent calculations were performed on the detrended smoothed profiles.

Pulse analysis. In order to quantify the pulsatile nature of insulin secretion and the relationship with variations in plasma glucose, each individual profile was submitted to ULTRA, a computer program for pulse detection and quantification [19]. The general principle of this algorithm is the elimination of all peaks for which either the increment (difference between the peak and the preceding trough) or the decrement (difference between the peak and the next trough) does not exceed a certain threshold related to measurement error. The output is a "clean" profile in which all temporal changes are significant with respect to assay precision. Simulation studies [19] have indicated that a threshold of twice the intra-assay coefficient of variation generally minimizes both false-positive and false-negative errors. However, because deconvolution involves an amplification of measurement error, a more conservative threshold of three times the intra-assay coefficient of variation of C-peptide has been previously used to quantify pulses of ISR $[5,6,9,11]$. Pulse analysis was performed on the profiles smoothed by the two-point moving average so that the measurement errors were divided by $\sqrt{2}$. Thus, peaks of insulin secretion and glucose were considered significant if their respective increments and decrements exceeded $8.49 \%$ (i.e. 3 times $4 \%$ divided by $\sqrt{2}$ ) and $2.12 \%$ (i.e. 2 times $1.5 \%$ divided by $\sqrt{2}$ ), respectively. For each significant pulse, the absolute amplitude was defined as the difference between the level at the peak and the level at the preceding trough. The relative amplitude was defined as the absolute amplitude, divided by the level of the preceding trough.

Because glucose has a relatively long half-life, changes in either production or utilization, or both, may not be reflected as significant peaks in the glucose concentration curve. As in previous studies $[5$, 11], we therefore identified all the "shoulders" in the "clean" glucose profiles. To do this, instantaneous derivatives of each individual "clean" glucose curve were estimated as the slope of the glucose changes during each 15-min sampling interval, and the shoulders were identified as slopes with an absolute value of zero, preceded and followed by slopes of the same sign.
Analysis of the temporal association between oscillations in glucose and ISR. Temporal associations between ultradian oscillations in glucose and ISR were quantified by pulse-by-pulse analysis of the concomitancy. Significant pulses of glucose and insulin secretion were considered concomitant if their peak values occurred within $15 \mathrm{~min}$ of each other or if their respective durations overlapped by more than $30 \mathrm{~min}$ or if both occurred $[5,6,9]$. The concomitance ratio of glucose pulses with ISR pulses was calculated as the number of concomitant glucose and ISR pulses divided by the total number of glucose pulses. The concomitance ratio of ISR pulses with glucose was calculated as the number of concomitant glucose and ISR pulses plus the number of concomitant glucose shoulders and ISR pulses, divided by the total number of ISR pulses.

Spectral analysis and distributions of interpulse intervals. To investigate the regularity of the recurrence of the ultradian oscillations in glucose concentrations and insulin secretion, the data were submitted to three different types of analyses. Theoretically, the oscillations could recur at fixed time intervals (i.e. the underlying generating process is strictly periodic), or at irregular time intervals but with some preferred frequency (i. e. the underlying generating process is pseudo-periodic) or be randomly distributed over time.

Each individual detrended profile of glucose and ISR was first submitted to a procedure designed to test the hypothesis that the temporal fluctuations can be considered as "white noise", i. e. purely random [20]. The alternative to the "white noise" hypothesis is the existence of periodic or pseudo-periodic components. This test is based on the fact that the integrated spectrum of a white noise process is a linear function of frequency and determines whether estimations of the integrated spectrum of the data in hand deviate significantly from a straight line when plotted against frequency.

Second, to identify possible dominating frequencies underlying the oscillations, estimations of the spectrum, i. e. the distribution of the variance of the profile over frequency, were obtained on each individual detrended glucose and ISR profile following Jenkins and Watts [20] using a Tukey window with a width of $6 \mathrm{~h}$ (i. e. 24 data points). The spectra were then averaged according to group.

Finally, all the interpulse intervals, i.e. the time intervals separating the peaks of successive significant pulses, were calculated for each of the series. The distribution of interpulse intervals was examined by plotting the percentage of the total number of interpulse intervals for each possible interpulse interval between $30 \mathrm{~min}$ (i. e. the shortest interpulse interval for data collected with a 15 -min sampling rate) and $420 \mathrm{~min}$ (i.e. the successive peaks are separated by $7 \mathrm{~h}$ ). With this procedure, the pattern of recurrence of the oscillations is examined independently of their amplitude.

Analysis of the profiles obtained at 2-min intervals. To eliminate the long-term trend associated with declining levels of glucose, C-peptide and insulin during prolonged fasting, the profiles obtained in the study with 2-min sampling were first detrended using the Cleveland algorithm [18] with a window of $7 \mathrm{~h}$. The ultradian oscillations were then characterized by a best-fit curve obtained using the Cleveland algorithm [18] with a window of $30 \mathrm{~min}$ (i. e. 15 sampling intervals). The amplitude of each ultradian oscillation was defined as the difference between the level at the peak of the best-fit curve and the level at the preceding trough. To quantify the rapid pulses independently of the slower oscillations, the best-fit curve was subtracted from the data and replaced by the mean level of all the data collected during the 8 -h study period. Pulses were identified using ULTRA [19] with a threshold for identification of twice the coefficient of variation of the assay.

\section{Statistical analysis}

Analysis of variance was performed to evaluate differences in the quantitative characteristics between the three subject groups. 
Table 1. Quantitative subject characteristics of the oscillations in glucose and insulin secretion rate (ISR) during fasting

\begin{tabular}{|c|c|c|c|c|}
\hline & $\begin{array}{l}\text { Type } 2 \\
\text { diabetic } \\
\text { patients } \\
(n=7)\end{array}$ & $\begin{array}{l}\text { Obese } \\
\text { control } \\
\text { subjects } \\
(n=8)\end{array}$ & $\begin{array}{l}\text { Normal } \\
\text { subjects } \\
(n=8)\end{array}$ & ANOVA \\
\hline \multicolumn{5}{|l|}{ Mean level } \\
\hline $\begin{array}{l}\text { Glucose }(\mathrm{mmol} / \mathrm{l}) \\
\text { Insulin (pmol/1) } \\
\text { ISR (pmol/min) }\end{array}$ & $\begin{array}{c}8.31 \pm 0.8^{\mathrm{a}, \mathrm{b}} \\
165 \pm 47^{\mathrm{a}, \mathrm{b}} \\
144.7 \pm 15.6^{\mathrm{a}, \mathrm{b}}\end{array}$ & $\begin{array}{l}4.82 \pm 0.11 \\
36 \pm 1 \\
91.8 \pm 12.0\end{array}$ & $\begin{array}{l}4.82 \pm 0.08 \\
36 \pm 6 \\
61.5 \pm 7.2\end{array}$ & $\begin{array}{l}p<0.001 \\
p<0.001 \\
p<0.001\end{array}$ \\
\hline \multicolumn{5}{|c|}{ Number of oscillations per $24 h$} \\
\hline $\begin{array}{l}\text { Glucose } \\
\text { pulses } \\
\text { shoulders } \\
\text { total } \\
\text { ISR pulses }\end{array}$ & $\begin{array}{r}6.7 \pm 0.7 \\
6.3 \pm 0.5 \\
13.0 \pm 1.0 \\
14.6 \pm 0.8\end{array}$ & $\begin{array}{r}8.4 \pm 0.7 \\
5.0 \pm 0.9 \\
13.4 \pm 0.9 \\
14.5 \pm 0.5\end{array}$ & $\begin{array}{r}6.0 \pm 1.4 \\
4.1 \pm 1.1 \\
10.1 \pm 1.3 \\
12.4 \pm 1.1\end{array}$ & $\begin{array}{l}\text { NS } \\
\text { NS } \\
\text { NS } \\
\text { NS }\end{array}$ \\
\hline \multicolumn{5}{|c|}{ Concomitancy ratio } \\
\hline $\begin{array}{l}\text { Glucose with ISR } \\
\text { ISR with glucose } \\
\text { pulses only } \\
\text { pulses and } \\
\text { shoulders }\end{array}$ & $\begin{array}{l}31 \pm 5 \% \\
64 \pm 7 \%\end{array}$ & $\begin{array}{l}45 \pm 3 \% \\
65 \pm 4 \%\end{array}$ & $\begin{array}{l}34 \pm 9 \% \\
63 \pm 8 \%\end{array}$ & $\begin{array}{l}\text { NS } \\
\text { NS }\end{array}$ \\
\hline \multicolumn{5}{|l|}{ Absolute amplitude } \\
\hline $\begin{array}{l}\text { Glucose }(\mathrm{mmol} / \mathrm{l}) \\
\text { ISR (pmol/min) }\end{array}$ & $\begin{array}{l}0.50 \pm 0.06^{\mathrm{a}, \mathrm{b}} \\
42.5 \pm 6.7^{\mathrm{a}}\end{array}$ & $\begin{array}{l}0.28 \pm 0.02 \\
27.8 \pm 3.2\end{array}$ & $\begin{array}{l}0.28 \pm 0.03 \\
15.9 \pm 1.5\end{array}$ & $\begin{array}{l}p<0.003 \\
p<0.001\end{array}$ \\
\hline \multicolumn{5}{|l|}{ Relative amplitude } \\
\hline $\begin{array}{l}\text { Glucose } \\
\text { ISR }\end{array}$ & $\begin{array}{r}6 \pm 0 \% \\
34 \pm 2 \%\end{array}$ & $\begin{array}{r}6 \pm 1 \% \\
39 \pm 3 \%\end{array}$ & $\begin{array}{r}6 \pm 1 \% \\
33 \pm 4 \%\end{array}$ & $\begin{array}{l}\text { NS } \\
\text { NS }\end{array}$ \\
\hline
\end{tabular}

a Significantly different from normal subjects with $p<0.05$ at least;

${ }^{b}$ significantly different from obese control subjects with $p<0.05$ at least;

'significantly different from obese control subjects by two-group Mann-Whitney test, $p<0.03$.

ANOVA, Analysis of variance

\section{Results}

\section{Mean levels and overall patterns of glucose and ISR}

Mean levels of plasma glucose, serum insulin and ISR during the fasting period are reported in Table 1 . The left panels of Figure 1 show representative raw data obtained in a patient with Type 2 diabetes. In both diabetic and nondiabetic subjects, glucose levels initially declined during fasting but subsequently rose in the later part of the night to peak in the early morning. ISR and serum insulin levels did not parallel glucose changes. A detailed analysis of these long-term trends has been reported elsewhere [13]. Pulses of glucose, insulin and ISR were apparent in all individual profiles in diabetic, obese and normal subjects. The right panels of Figure 1illustrate how smoothing and detrending improved the detection of pulses. As exemplified in this subject, the profiles of serum insulin closely paralleled the profiles of ISR. However, during fasting, serum insulin levels were frequently in the lower range of assay limits and therefore estimations of Beta-cell secretory activity were more accurate when based on the ISR profiles derived from the C-peptide levels. Thus, all analyses were based on the ISR, rather than serum insulin, profiles.

\section{Pulse analysis of glucose and ISR profiles}

As suggested by visual examination of Figure 1, pulses of ISR appeared to be more frequent than glucose pulses. This was confirmed by formal pulse identification. Indeed, there were approximately $12-15$ ISR pulses per $24-\mathrm{h}$ period as compared to 6-9 glucose pulses (Table 1 ). The frequency of ISR and glucose pulses during fasting did not differ significantly between Type 2 diabetic patients, obese patients and normal subjects. Patients with Type 2 diabetes tended to have fewer glucose pulses than non-diabetic control subjects $(6.7 \pm 0.7$ vs $8.4 \pm 0.7, p<0.10)$. A greater proportion of the total number of glucose oscillations were reflected as shoulders rather than as discrete peaks in diabetic subjects than in non-diabetic control subjects. Indeed, $48 \%$ of the total number of glucose oscillations corresponded to shoulders in diabetic subjects as compared to $37 \%$ in nondiabetic control subjects.

\section{Temporal relationship between oscillations of glucose and ISR}

Analysis of concomitancy revealed that, on average, 65 $79 \%$ of glucose pulses were temporally associated with an ISR pulse but that less than half of the ISR pulses were concomitant with a significant glucose pulse (Table 1). However, when shoulders in the glucose profiles were taken into account in the concomitancy analysis, it appeared that approximately two-thirds of the ISR pulses were associated with a significant change in glucose levels.

When expressed in absolute levels, pulse amplitudes for both glucose and ISR were higher in Type 2 diabetic patients than in non-diabetic subjects (Table 1). In each individual, the mean absolute amplitude of pulses was directly related to the $24-\mathrm{h}$ mean level for both glucose and ISR. Indeed, the coefficient of correlation between mean pulse amplitude and 24-h mean level was 0.893 for glucose and 0.926 for ISR $(p<0.01)$. Thus, increased levels of glucose and insulin secretion are associated with increased amplitudes of oscillation. When expressed as percentage increase over the preceding trough, pulse amplitudes averaged $6 \%$ for glucose in all three groups of subjects. Relative pulse amplitudes for ISR were also similar in the three groups of subjects, averaging approximately $35 \%$ (Table 1).

\section{Spectral analysis of the glucose and ISR profiles}

For each of the constituent series, the hypothesis that the oscillations might represent random biological noise rather than contain a temporal signal including significant contributions from periodic or pseudo-periodic components was rejected at a probability level of $p<0.05$.

Spectral analysis further delineated the characteristics of the oscillations in insulin secretion and glucose levels during fasting in normal volunteers, obese subjects and diabetic patients. As may be seen in Figure 2, the mean spectra for ISR were similar in the three groups of subjects with $75 \%$ of the total variance being confined to the period range $60-360 \mathrm{~min}$, reflecting the inter-individual dif- 

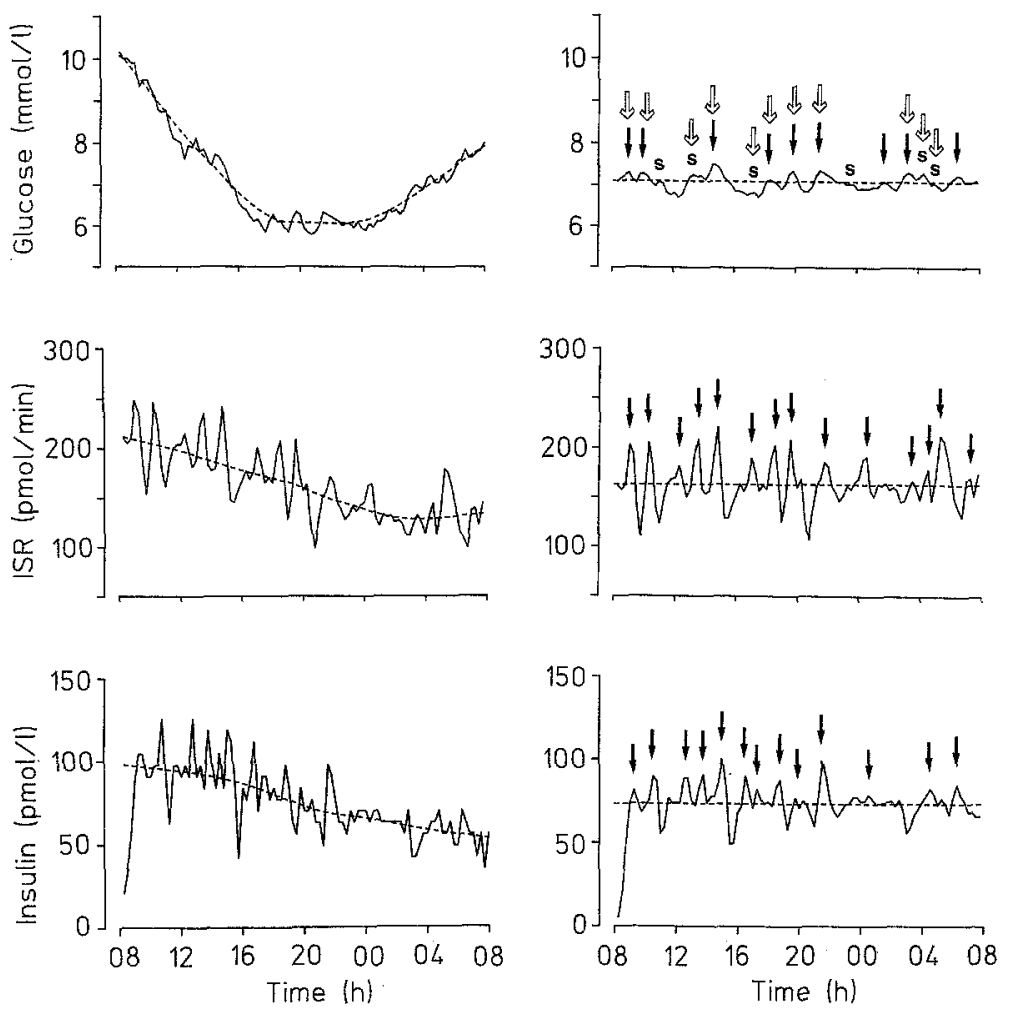

Fig. 1. The left panels show the raw data for plasma glucose levels (top), insulin secretory rates (ISR; middle) and serum insulin (bottom) in a Type 2 (non-insulindependent) diabetic patient. The dashed lines represent the best-fit curve quantifying the long-term trend. The right panels show the detrended smoothed profiles. Short-term oscillations of glucose, serum insulin and ISR are now clearly apparent. The dashed line represents the 24-h mean level. Significant pulses are indicated by solid arrows. Glucose shoulders are indicated by "S". Open arrows indicate the pulses and shoulders in plasma glucose which were concomitant with a significant pulse in ISR ferences in average recurrence time of ISR pulses as well as persistence of slower variations not eliminated by the detrending procedure. While the differences in spectra of ISR oscillations between diabetic patients, obese control and normal subjects were small, marked differences between normal subjects, on the one hand, and obese and diabetic patients, on the other hand, were readily apparent for the glucose spectra. Indeed, in normal subjects, the majority of the variance occurred in the $100-180 \mathrm{~min}$ range. However, in the diabetic patients, there was essentially no power associated with periods shorter than $144 \mathrm{~min}$, as the majority of the variability was confined in the low frequency range, corresponding to periods of $180 \mathrm{~min}$ and longer. These differences in the spectra indicate that glucose oscillations were generally slower in the Type 2 diabetic patients than in normal subjects and do not present a dominant ultradian periodicity in the 100 180 min range. In obese subjects, the glucose spectrum also showed a displacement towards the low frequency range as compared to normal subjects, with the spectrum dominated by periods of 180-360 min. However, the shift was less pronounced than in the diabetic patients and temporal variability with periods below $144 \mathrm{~min}$ was not as greatly suppressed. Thus, in both Type 2 diabetic patients and obese control subjects, there was a partial dissociation between the glucose spectrum and the ISR spectrum with the majority of spectral power for glucose confined to the period range above $144 \mathrm{~min}$ while ISR showed significant spectral power associated with periods shorter than $144 \mathrm{~min}$. In contrast, in normal subjects, the overlap between the glucose spectrum and the ISR spectrum extended from the lower limit of the frequency range to periods of approximately $90 \mathrm{~min}$. At higher frequencies (i.e. shorter periods), there were still large power estimates in the ISR spectrum but not in the glucose spectrum. This confirmed the findings from the pulse analysis indicating that ISR pulses are more frequent than glucose pulses.

\section{Distributions of interpulse intervals}

The characteristics of the distributions of interpulse intervals shown in Figure 3 are in agreement with the results from the spectral analysis. Indeed, for ISR, the distribution of interpulse intervals is remarkably similar in the three groups but, for glucose, a shift towards the long interpulse intervals (i.e. low frequency range) is apparent in diabetic patients as compared to non-diabetic subjects. Indeed, in diabetic subjects, interpulse intervals of $180 \mathrm{~min}$ and longer represent $60 \%$ of the total number of glucose interpulse intervals whereas, in normal and obese subjects, the corresponding proportions are $32 \%$ and $45 \%$, respectively. In the diabetic patients, this results in a marked dissociation between the distribution of interpulse intervals for ISR and for glucose, with only $6 \%$ of intervals of $180 \mathrm{~min}$ or longer for ISR in comparison to $60 \%$ for glucose. In non-diabetic subjects, there is a much closer concordance between the distributions of interpulse intervals for ISR and glucose.

\section{Relationship between ultradian oscillations and rapid 10-15 min periodicity}

Figure 4 shows the profiles of plasma glucose, plasma Cpeptide and serum insulin sampled at 2-min intervals during an 8-h fast. Rapid oscillations are clearly superim- 

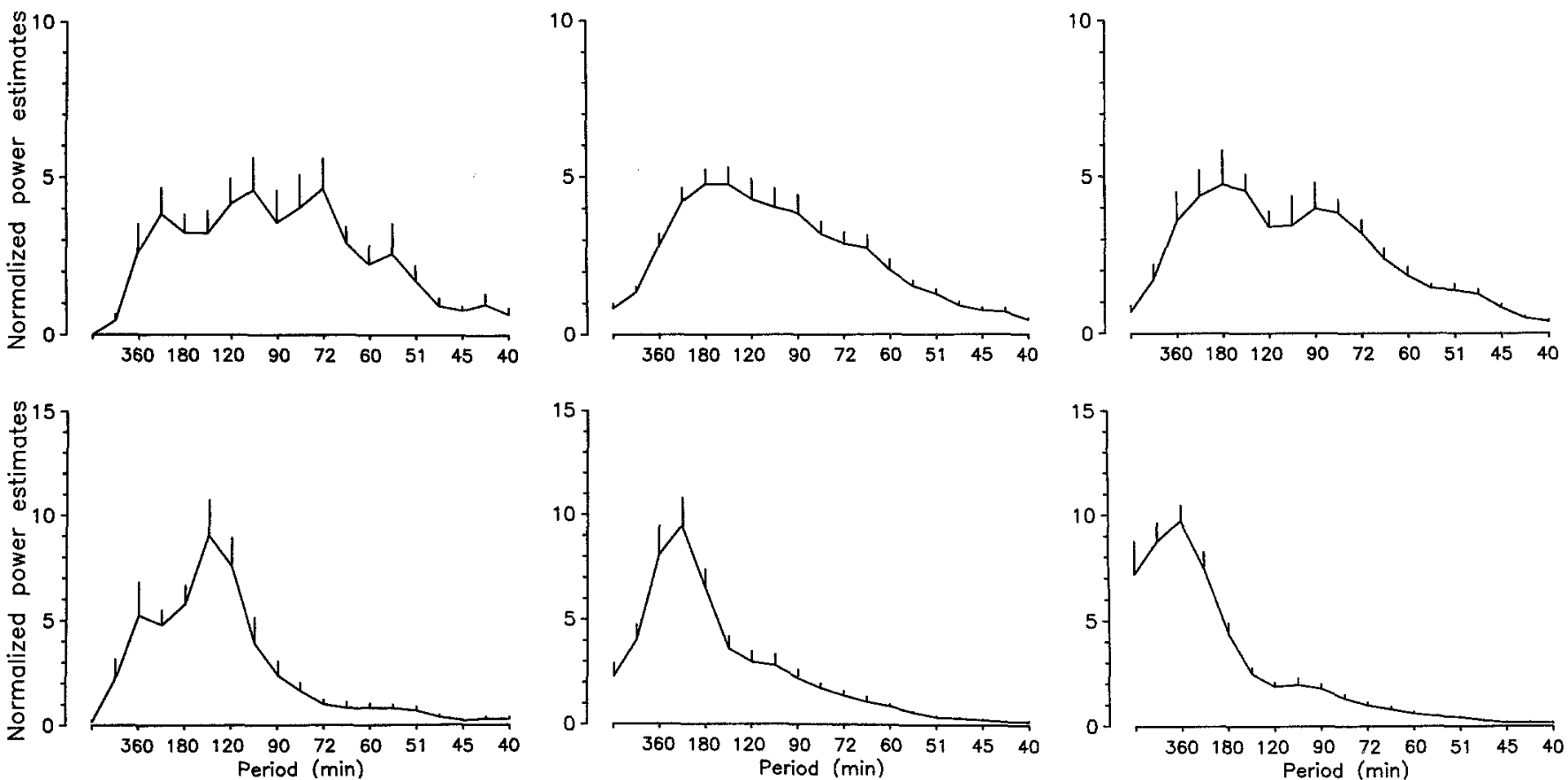

Fig. 2. Normalized smoothed spectral estimates for insulin secretion rate (ISR, top) and glucose (bottom) in normal subjects (left), nondiabetic obese subjects (centre) and patients with Type 2 (non-insulin-dependent) diabetes mellitus (right). Mean spectra across each group are shown and the vertical bars represent SEM. If the spectral estimates were not normalized, the areas under the curves would be equal to the total variance of the original series, which is a measure

of its temporal variability. Normalizing the spectral estimates implies that the variance of the series is considered to be $100 \%$ and the calculated spectrum then represents the relative distribution of the variance over frequency or period. Note the shift of the glucose spectrum towards slower periods in diabetic patients as compared to normal subjects. This is in contrast to the ISR spectra which cover essentially the same period range in the three groups of subjects

posed on slower trends. These include a progressive decline in overall levels of glucose, C-peptide and insulin as the fast is prolonged (Fig. 4, left panels), and slow oscillations in the ultradian range, which are more clearly revealed when the long-term trend is removed (Fig. 4, right panels). Six synchronous ultradian oscillations of C-peptide and insulin are apparent, corresponding to a periodicity of $80 \mathrm{~min}$. Five of these oscillations are paralleled by similar glucose changes. The relative amplitude of the ultradian oscillations averaged $16 \pm 6 \%$ for C-peptide and $23 \pm 17 \%$ for insulin (mean \pm SD) .

Rapid oscillations were particularly prominent for insulin, with 43 significant pulses being identified over the 8 $\mathrm{h}$ study period (i.e. one pulse every $11.2 \mathrm{~min}$ ), and a mean relative amplitude of $37 \pm 19 \%$ (mean \pm SD). For C-peptide, the rapid oscillations were of smaller magnitude, and thus fewer of them met the criteria for significance: a total of 36 pulses were detected (i.e. one pulse every $13.3 \mathrm{~min}$ ) with a relative amplitude of $14 \pm 7 \%$ (mean \pm SD). Rapid glucose oscillations were less frequent, with 17 significant pulses over the 8-h study period (i.e. one pulse every $28.2 \mathrm{~min}$ ), and their relative amplitude averaged $4 \pm 1 \%$.

\section{Discussion}

The present study was undertaken to explore the dynamic temporal interactions between insulin secretion rates and plasma glucose concentrations in patients with Type 2 diabetes, control subjects matched for degree of obesity and normal volunteers. The results show that, even under fasting conditions, insulin secretion and plasma glucose levels are characterized by a series of non-random ultradian oscillations. This ultradian periodicity appears to be independent of the rapid 10-15 min pulses and rapid sampling for prolonged periods of time clearly revealed the co-existence of both oscillatory processes. The diabetic state is associated with a slowing of the ultradian oscillations in glucose, without change in the frequency of the oscillations in insulin secretion.

These findings were obtained using two independent mathematical approaches to quantify the temporal patterns of changes in insulin secretion and glucose levels. The first approach was an analysis in the time domain involving the individual identification of each oscillation or "pulse". From this "time domain" analysis, the relationships between overall mean levels and pulse amplitudes were derived and the patterns of recurrence of the oscillations were examined by calculating the distributions of interpulse intervals. The second approach was an analysis in the frequency domain and included testing whether the oscillations could represent purely random noise and estimating the distribution of temporal variability over frequency, i.e. the spectrum. These two independent approaches concurred in showing that the ultradian oscillations of insulin secretion and glucose levels which had been previously observed in conditions of stimulated pancreatic function $[3-9,11]$, persist during the fasting state 

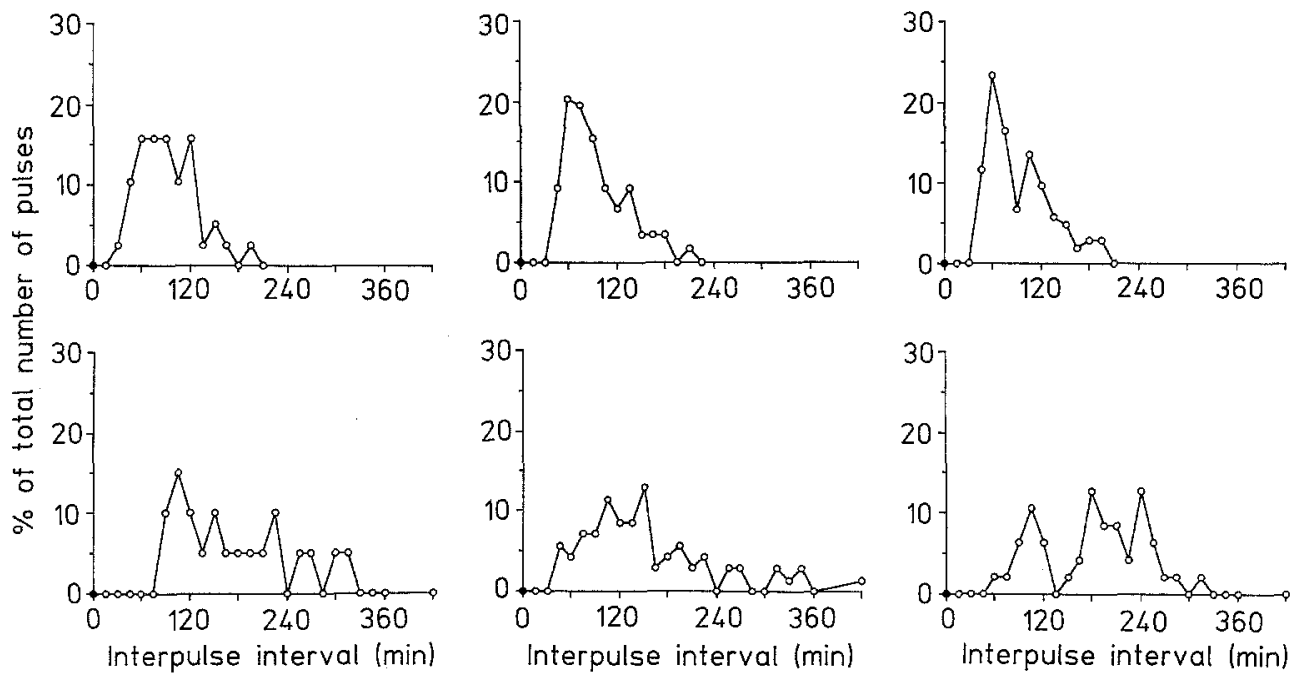

Fig.3. Distribution of interpulse intervals for insulin secretion rate (ISR, top) and glucose (bottom) in normal subjects (left), non-diabetic obese subjects (centre) and patients with Type 2 (non-insulin-dependent) diabetes mellitus (right). Note the similarity in the distributions of interpulse intervals for ISR in the three groups and the shift towards longer interpulse intervals for glucose in the diabetic group
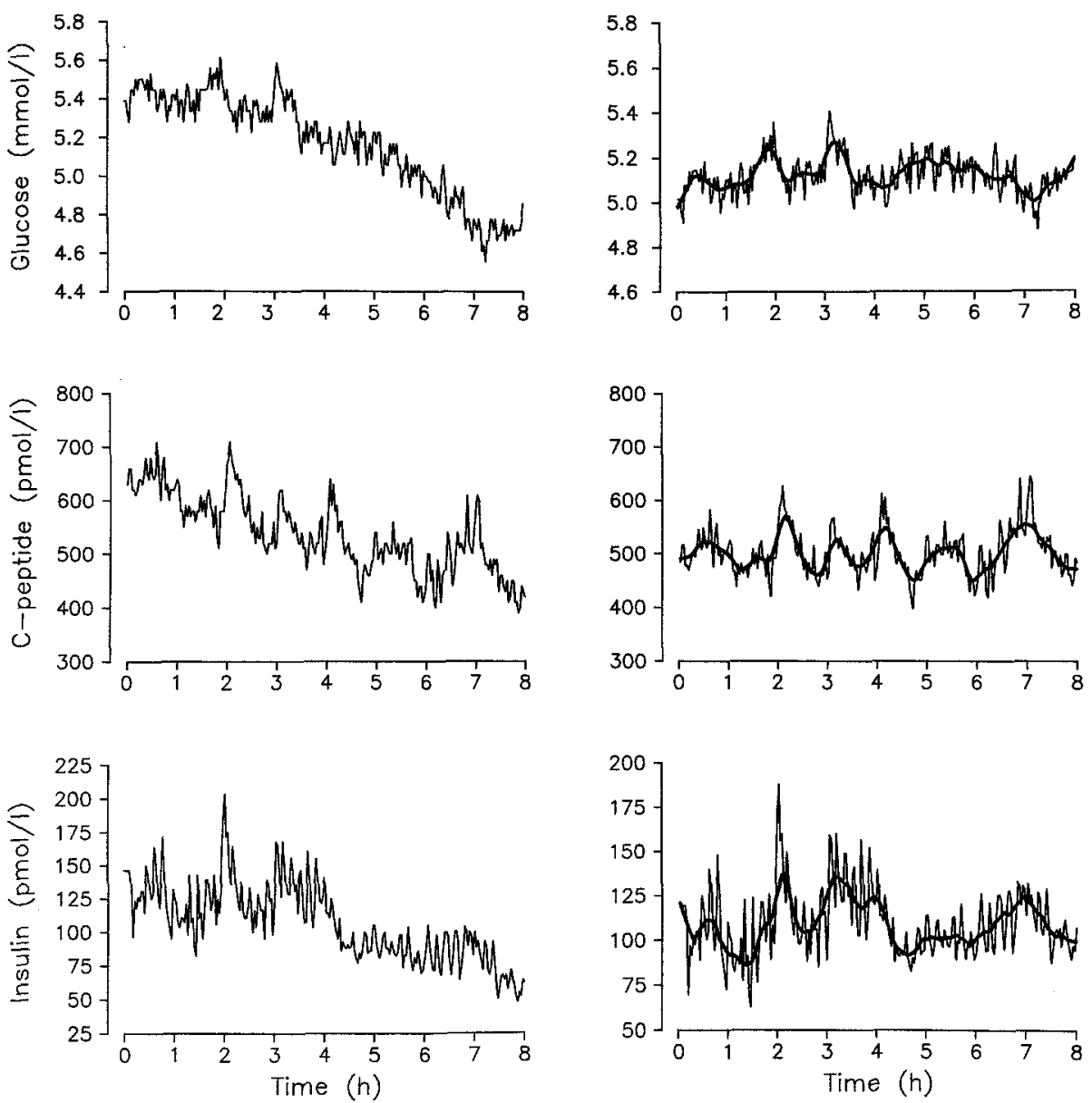

Fig.4. Profiles of plasma glucose (top), plasma C-peptide (centre) and serum insulin (bottom) obtained at 2-min intervals during an 8 -h period of fasting, starting at 08.00 hours, following an overnight fast. The left panels show the raw data, with the expected declining trend in overall glucose, C-peptide and insulin levels. The right panels show the detrended data. The rapid 10-15 min oscillations appear superimposed on the slower ultradian oscillations which are evidenced by a best-fit curve obtained by a regression algorithm

and indicate that some of the abnormalities in the patterns of oscillations which had been found postprandially in Type 2 diabetic patients [9] may also be detected during prolonged fasting.

The results have allowed a number of features of the insulin secretory profile to be defined under fasting conditions. In particular, both in diabetic and non-diabetic subjects, the relative amplitude of the oscillations in insulin secretion was 5-6 fold greater than the relative amplitude of the glucose oscillations. This difference in relative am- plitude of the oscillations probably relates to the fact that insulin secretion is almost completely inhibited when glucose concentrations reach the hypoglycaemic range, and thus there is a relatively higher constant background level for glucose than for ISR. The finding is also consistent with the hypothesis that the secretory oscillations are necessary to maintain the plasma glucose levels within a narrow range. The amplitude of the oscillations in both ISR and glucose was significantly correlated with their respective mean levels and thus the absolute amplitudes of 
both the ISR and glucose oscillations were greater in the diabetic patients than in the control subjects. However, the relative amplitude of both glucose (approximately $6 \%$ ) and ISR oscillations (approximately 35\%) was similar in diabetic and non-diabetic subjects.

The patterns of oscillations in insulin secretion were remarkably similar in normal, obese and diabetic subjects, with $12-15$ oscillations occurring over the $24-h$ cycle. This estimation of the frequency of oscillations in insulin secretion is essentially identical to that obtained during constant glucose infusion $[5,7]$, suggesting that the same oscillatory process is operative under stimulated and unstimulated conditions. We were also unable to demonstrate significant differences between diabetic and nondiabetic subjects in the patterns of recurrence of the secretory oscillations, whether quantified by spectral analysis or by calculation of the distributions of interpulse intervals. Spectral analysis did not, however, delineate a narrow range of preferred periods of recurrence for the insulin secretory oscillations. This unexpected result may in part reflect the undersampling of the rapid oscillations, which could have caused some distortion of the spectral estimations due to aliasing [20], a phenomenon which occurs when a signal including high frequency components is sampled at a rate too low to estimate these rapid changes. As demonstrated by the 8-h study with 2-min sampling, ultradian oscillations and rapid pulses both contribute to the complex temporal pattern of insulin secretion and, during fasting, these periodicities are of similar amplitude. Therefore, in the studies with sampling at 15-min intervals, aliasing effects may have resulted in a loss of precision in the characterization of individual pulses and in a distortion of the spectral estminations. Another possible explanation for the lack of dominant peak in the ISR spectrum is that, in the fasting state, secretion is relatively more affected by glucose-independent factors which may contaminate the oscillatory signal.

As has been previously observed during constant glucose infusion $[5,7,11]$, the number of oscillations per $24-\mathrm{h}$ is significantly less for glucose than for ISR. Moreover, the number of significant glucose pulses tended to be less in diabetic subjects than in the control subjects. The slowing of the glucose oscillations in diabetic subjects was clearly evidenced by the examination of the distribution of interpulse intervals and of the spectral analysis. Indeed, both approaches showed a displacement of the rate of recurrence of the oscillations towards the low frequency range, corresponding to longer interpulse intervals. The broader, more sluggish oscillations of plasma glucose without sharp peaks seen in Type 2 diabetes could result from a failure of insulin to suppress either hepatic glucose production or the presence of peripheral insulin resistance, or both, resulting in diminished glucose utilization.

The slowing of the glucose oscillations, without significant change in frequency of the ISR oscillations, could be interpreted as a partial uncoupling of the glucose oscillations from the ISR oscillations in Type 2 diabetes. However, it is noteworthy that, when glucose shoulders are included in the analysis of concomitancy, the number of ISR oscillations which occur in association with glucose changes becomes similar in diabetic and non-diabetic sub- jects. It is conceivable that, if tracer techniques were used in an attempt to separate terms of glucose production and glucose utilization, a tighter association between oscillations in insulin secretion and oscillations in either glucose production or glucose utilization would emerge. The reduction in concomitancy rates in diabetic patients is also compatible with a slower clearance of glucose in these people.

The present data have some implications for our recently published model [12] for the mechanisms generating ultradian oscillations in non-diabetic subjects. The substantial slowing of the glucose oscillation without a corresponding reduction in the ISR period in Type 2 diabetes suggests that factors other than glucose may be involved in generating and regulating the oscillations in conditions of glucose intolerance. Such factors may be secondary to glucose in normal subjects but of relatively more importance in Type 2 diabetes. These may include other insulin secretagogues such as arginine and glucagon. Furthermore, it is quite certain that the sensitivity of the secretory output of the Beta cell to glucose is more variable and complex than the simple sigmoidal relationship assumed in our model, in particular in diabetes where the Beta-cell responsiveness to glucose is likely to be impaired. In addition, the functional relationships for glucose utilization and glucose production assumed in the model were characteristic of subjects with no insulin resistance and would therefore have to be adjusted to account for the diabetic condition. Further simulation studies on modified models could facilitate the identification of factors associated with abnormalities of the ultradian oscillations in the diabetic state.

A comparison of the present results obtained during prolonged fasting with previously published data in which oscillations were studied following stimulation by mixed meals and continuous enteral nutrition $[6,9,21]$, reveals both similarities and differences in the properties of the oscillations under stimulated and non-stimulated conditions in diabetic and non-diabetic subjects. During fasting conditions as well as following meal ingestion, the glucose oscillations were more sluggish in the diabetic patients than in the control subjects [9]. This observation is in contrast to the situation observed during enteral nutrition where the oscillatory glucose patterns in diabetic patients were similar to those observed in non-diabetic subjects [21]. We have observed similar fasting patterns of ISR between the three groups in the present study. However, following meals, there was a difference in amplitude of the secretory oscillations between diabetic and non-diabetic subjects, with a reduction by more than $50 \%$ of the amplitude of the ISR oscillation in diabetic patients as compared to non-diabetic control subjects [9], and during enteral nutrition, the ISR oscillations exhibited an altered periodicity in the diabetic patients [21]. Finally, the strengthening of the oscillatory signal associated with the stimulated condition resulted, in non-diabetic subjects, in a tighter coupling between oscillations in insulin secretion and of glucose, evidenced by higher concomitancy rates between ISR pulses and glucose pulses. Indeed, the concomitancy rate of ISR with glucose averaged only $65 \%$ during fasting whereas following meal ingestion and dur- 
ing enteral nutrition this ratio was above $80 \%[9,21]$. However, in the diabetic patients, despite increases in relative amplitude of both the glucose and ISR oscillations, the concomitancy ratio following meal ingestion or during enteral nutrition was not higher than during fasting $[9,21]$. Thus, in diabetes, abnormalities in the ultradian oscillations of insulin secretion and glucose are more pronounced during the stimulated state than under basal conditions. Further studies are needed to determine if subtle abnormalities in the ultradian oscillations appear during fasting at an early stage in the development of Type 2 diabetes and if these oscillations play a role in the pathogenesis of the disorder.

Acknowledgements. This study was supported in part by grants DK31842, DK-20595 and DK-41814 from the National Institutes of Health and the General Clinical Research Center at the University of Chicago (RR-00055). We thank the volunteers for their participation in these studies and the nursing staff of the Clinical Research Center for their skillful assistance.

\section{References}

1. Lang DA, Matthews DR, Phil D, Peto J, Turner RC (1979) Cyclic oscillations of basal plasma glucose and insulin concentrations in human beings. N Engl J Med 301: 1023-1027

2. Hansen BC, Jen KL, Pek SB, Wolfe RA (1982) Rapid oscillations in plasma insulin, glucagon, and glucose in obese and normal weight humans. J Clin Endocrinol Metab 54: 785-792

3. Kraegen EW, Young JD, George EP, Lazarus L (1972) Oscillations in blood glucose and insulin after oral glucose. Horm Metab Res 4: 409-413

4. Simon C, Brandenberger G, Follenius M (1987) Ultradian oscillations of plasma glucose, insulin, and C-peptide in man during continuous enteral nutrition. J Clin Endocrinol Metab 64: 669 674

5. Shapiro ET, Tillil H, Polonsky KS, Fang VS, Rubenstein AH, Van Cauter E (1988) Oscillations in insulin secretion during constant glucose infusion in normal man: relationship to changes in plasma glucose. J Clin Endocrinol Metab 67: 307-314

6. Polonsky KS, Given BD, Van Cauter E (1988) Twenty-four-hour profiles and pulsatile patterns of insulin secretion in normal and obese subjects. J Clin Invest 81: 442-448

7. Van Cauter E, Desir D, Decoster C, Féry F, Balasse EO (1989) Nocturnal decrease of glucose tolerance during constant glucose infusion. J Clin Endocrinol Metab 69: 604-611
8. Simon C, Follenius M, Brandenberger G (1987) Postprandial oscillations of plasma glucose, insulin and C-peptide in man. Diabetologia 30: 769-773

9. Polonsky KS, Given BD, Hirsch LJ et al. (1988) Abnormal patterns of insulin secretion in non-insulin-dependent diabetes mellitus. N Engl J Med 318: 1231-1239

10. Marsh BD, Marsh DJ, Bergman RN (1986) Oscillations enhance the efficiency and stability of glucose disposal. Am J Physiol 250: E576-E582

11. Sturis J, Van Cauter E, Blackman JD, Polonsky KS (1991) Entrainment of pulsatile insulin secretion by oscillatory glucose infusion. J Clin Invest 87: 439-445

12. Sturis J, Polonsky KS, Mosekilde E, Van Cauter E (1991) Computer model for mechanisms underlying ultradian oscillations of insulin and glucose. Am J Physiol 260: E801-E809

13. Shapiro ET, Polonsky KS, Copinschi G et al. (1991) Nocturnal elevation of glucose levels during fasting in noninsulin-dependent diabetes. J Clin Endocrinol Metab 72:444-454

14. Eaton RP, Allen RC, Schade DS, Erickson KM, Standefer J (1980) Prehepatic insulin production in man: kinetic analysis using peripheral connecting peptide behavior. J Clin Endocrinol Metab 51:520-528

15. Polonsky KS, Licinio-Paixao J, Given BD et al. (1986) Use of biosynthetic human $\mathrm{C}$-peptide in the measurement of insulin secretion rates in normal volunteers and type I diabetic patients. $J$ Clin Invest 77: 98-105

16. Morgan C, Lazarow A (1963) Immunoassay of insulin: two antibody systems: plasma insulin levels of normal, subdiabetic and diabetic rats: Diabetes 12: 115-126

17. Faber OK, Binder C, Markussen J et al. (1978) Characterization of seven C-peptide antisera. Diabetes 27 [Suppl 1]: 170-177

18. Cleveland W (1979) Robust locally weighted regression and smoothing scatterplots. J Am Stat Assoc 74: 829-836

19. Van Cauter $\mathrm{E}$ (1988) Estimating false-positive and false-negative errors in analyses of hormonal pulsatility. Am J Physiol 254: E786-E794

20. Jenkins GM, Watts DG (1968) Spectral analysis and its application. Holden Day, San Francisco

21. Simon C, Brandenberger G, Follenius M, Schlienger JL (1991) Alteration in the temporal organisation of insulin secretion in Type 2 (non-insulin-dependent) diabetic patients under continuous enteral nutrition. Diabetologia 34: 435-440

Received: 11 November 1991

and in revised form: 1 April 1992

Dr. E. Van Cauter

Department of Medicine, MC 1027

5841 S. Maryland Avenue

Chicago, IL 60637

USA 